\title{
Low melting temperature and liquid surface layering for pair potential models
}

\author{
E. Velasco and P. Tarazona \\ Departamento de Física Teórica de la Materia Condensada, Universidad Autónoma de Madrid, \\ E-28049 Madrid, Spain and Instituto Nicolás Cabrera, Universidad Autónoma de Madrid, \\ E-28049 Madrid, Spain \\ M. Reinaldo-Falagán \\ Departamento de Física Teórica de la Materia Condensada, Universidad Autónoma de Madrid, \\ E-28049 Madrid, Spain \\ E. Chacón ${ }^{\text {a) }}$ \\ Instituto de Ciencia de Materiales de Madrid, Consejo Superior de Investigaciones Científicas, \\ Cantoblanco, E-28049 Madrid, Spain
}

(Received 3 July 2002; accepted 20 September 2002)

\begin{abstract}
We have recently proposed [Phys. Rev. Lett. 87, 166101 (2001)] that any isotropic fluid should exhibit surface layering at its liquid-vapor interface above the triple temperature provided that the system has a low triple temperature. In this article we present an extensive study of systems with different isotropic pair interactions, some of which present a very low triple temperature. We have confirmed that surface layering is a general characteristic of very cold liquids, independent of the specific shape of the potential, and that only pair potentials presenting a low triple-point temperature do exhibit surface oscillations; in other cases layering is preempted by solidification. Finally, we study the damping of surface oscillations due to capillary waves and conclude that for any model pair potential the temperature threshold below which layering would be observed for the typical experimental transverse sampling sizes is $15 \%$ of the critical temperature. (c) 2002 American Institute of Physics. [DOI: 10.1063/1.1521160]
\end{abstract}

\section{INTRODUCTION}

Recent x-ray reflectivity measurements have verified the existence of oscillations along the normal to the liquidvapor interface for metals, $\mathrm{Hg}^{1}$ and $\mathrm{Ga}^{2}{ }^{2}$ with low melting temperature. Although liquid surface layering is well-known in anisotropic fluids (for example liquid crystals ${ }^{3,4}$ ) and at the interface between a simple fluid and a sharp wall, ${ }^{5}$ it was believed that layering is absent at the liquid surface of simple fluids with isotropic interactions. ${ }^{6,7}$ No $x$-ray reflectivity measurements have been reported for elemental metals with higher melting temperature, for example, the alkali metals. However Tostmann et al. ${ }^{8}$ have recently measured the surface structure of the eutectic $\mathrm{K}_{67} \mathrm{Na}_{33}$ mixture, which exhibits a bulk liquid structure factor characteristic of an ideal mixture and therefore can be expected to share some properties of elemental liquid alkali metals. While less pronounced than in the case of $\mathrm{Hg}$ and $\mathrm{Ga}$, the results of Tostmann et al. indicate the presence of surface-induced layering. Simulations ${ }^{9,10}$ and theoretical ${ }^{11}$ studies suggested that surface layering in these systems is due to the metallic character of the binding interactions, and that the many-body effects arising from delocalized electrons give rise to the appearance of surface oscillations. In the case of $\mathrm{Ga}$ it has also been suggested that the relatively high degree of covalent bonding could be responsible for the presence of oscillations. $^{2,12}$

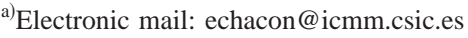

In a recent letter ${ }^{13}$ we have argued that layering at free liquid surfaces is a more universal behavior, and that the many-body nature of metallic interactions may not necessarily play a leading role. Fluids with isotropic pair interactions exhibit oscillatory interfacial structures, for temperatures close to the melting temperature, provided that the ratio between the melting $\left(T_{m}\right)$ and critical $\left(T_{c}\right)$ temperatures is low. Our conclusion is that layering should be a common feature of cold liquid surfaces well below the critical temperature, but that in simple molecular fluids it is preempted by solidification, below which the liquid becomes metastable and the free liquid-vapor interface cannot be observed at equilibrium. This result agrees with the fact that the two systems where layering has been clearly observed, $\mathrm{Hg}$ and $\mathrm{Ga}$, both have low melting points, with a ratio $T_{m} / T_{c}=0.13$ for $\mathrm{Hg}$ and even lower for Ga. Other liquid metals such as the alkali have a ratio close to 0.15 which, despite being much lower than the corresponding value $(0.55)$ for the noble gases, would be at the limit of observation. ${ }^{8}$

The aim of the present paper is to analyze several simple pair molecular interactions in order to elucidate the relation between the shape of the effective pair potential and the existence of a low $T_{m} / T_{c}$ ratio. Based on a study of effective pair potentials typical of dense metallic fluids, we have identified two mechanisms that can be responsible for a lowering of the melting temperature (obviously, other mechanisms cannot be ruled out). The first is the presence of soft repulsions, whereas the second mechanism is associated with a 
TABLE I. Parameters for several effective pair potentials within the Na parametric family.

\begin{tabular}{lccccc}
\hline \hline Potential & $A_{0}(\mathrm{eV})$ & $\alpha\left(\AA^{-1}\right)$ & $A_{1}(\mathrm{eV})$ & $\beta\left(\AA^{-1}\right)$ & $R_{1}(\AA)$ \\
\hline Na & 437.96 & 2.2322 & 0.18382 & 0.2140 & 3.5344 \\
soft-1 & 37.550 & 1.4881 & 0.21054 & 0.2140 & 3.5344 \\
soft-2 & 68.247 & 1.6741 & 0.20015 & 0.2140 & 3.5344 \\
repul & 947523 & 4.4644 & 0.16684 & 0.2140 & 3.5344 \\
\hline \hline
\end{tabular}

broad and flat attractive well with a width that destabilizes solid compact structures such as the face-centered cubic (fcc) and hexagonal close-packed (hcp) lattices. In this work we have analyzed two families of potentials, one for each of the traits mentioned above. In the first family, which we name alkalilike potentials, we have considered a number of different potentials having soft repulsive cores. The second, called mercurylike potentials, includes model potentials with harsh repulsive cores and flat, wide attractive wells.

One of the aims of this paper is to present a detailed treatment that improves and extends our recent letter. Also, we present an alternative and more accurate methodology to locate the triple point of the different systems, based on directly calculating the free energies of the phases involved by means of thermodynamic integration, instead of the bulk isobaric and direct interface Monte Carlo (MC) simulations used previously. We present clear evidence that in all cases where the systems have a low $T_{t} / T_{c}$ the corresponding liquid-vapor interfaces exhibit oscillations at temperatures close to $T_{t}$. In order not to make the presentation too extensive, a detailed analysis of the properties of the corresponding density profiles is not included. The latter study, which includes topics such as capillary-wave effects, dependence of layering on system size, and connection with the FisherWidom line, is presented elsewhere. ${ }^{14}$

Despite being a demanding technique from the computational point of view, all the results presented in this paper have been obtained by means of computer simulations. It is well known that there is a lack of suitable analytical techniques based on density-functional (DF) approximations to study strongly correlated fluids with soft potential interactions. Usually, for hard-core potentials, details such as the choice of a reference system for a perturbative treatment of the molecular attractions or of a weighted-density approximation for the treatment of the hard core, do not change qualitatively the predictions of different density-functional approximations for the liquid-vapor or fluid-wall interfaces. However, for the potentials analyzed in this work, all of which possess soft cores, we have found that the predictions for $T_{t}$ and for the oscillatory behavior of the longitudinal density distribution (density profile) $\rho(z)$ can be easily shuffled with different reasonable approximations for $F[\rho]$. The low melting temperatures are linked to the presence of strong correlations in the liquid, and the subtle thermodynamic balance with the crystal phase becomes too difficult to analyze with DF theories. Our opinion is that, with the present state of the art, the theory does not give a conclusive answer to our question. Thus, we decided to focus our work on the results of the MC simulations, leaving out interesting but quite different issues on the validity of the usual DF approximations.

In the next section a discussion on the melting properties and liquid-vapor equilibria of the various pair potentials is presented; we consider in turn the two families of potentials, the alkalilike and mercurylike families, and make a parallel presentation for the two cases. The surface properties of the potentials are analyzed in Sec. III which includes a discussion on the effect of capillary waves on the oscillatory surface structures. We finish in Sec. IV with a summary of our work and conclusions.

\section{SYSTEMS WITH LOW MELTING TEMPERATURE}

\section{A. The alkalilike systems}

\section{Pair potentials}

In order to find pair potentials with a low reduced melting temperature we started with effective-model descriptions reproducing some features of the alkali metals. We used total energy local density approximation results for $\mathrm{Na}$ crystals, calculated as a function of the lattice constant for both bcc and fcc lattices, ${ }^{15}$ and searched for the best fit to an additive pair-potential interaction $E=\Sigma_{i, j} \phi\left(r_{i j}\right)$, within a broad parametrized functional family for $\phi(r)$. Inverse power forms, including the Lennard-Jones (LJ) potential, could not fit crystal energies to that simple pairwise form, but an accurate fit can be obtained using

$$
\phi_{\mathrm{Na}}(r)=A_{0} e^{-\alpha r}-A_{1} e^{-\beta\left(r-R_{1}\right)^{2}},
$$

where the exponential term describes mainly the repulsive part of the potential and the attraction is given by the Gaussian function. The values of the parameters that provide the best fit are given in Table I. The resulting pair potential, called henceforth $\phi_{\mathrm{Na}}(r)$ and shown in Fig. 1, is quite different from a LJ potential $\phi_{\mathrm{LJ}}(r)$-much sharper in the repulsive part and narrower in the attractive well. The soft repulsion of our effective potential agrees with the general characteristics of alkali metal interactions ${ }^{16,17}$ where the softness of the repulsion reflects the overlap between open valence levels, as opposed to closed valence orbitals as in noble gases or molecular fluids. Note that in order to compare the different models reduced units are defined in terms of a length $\sigma$ that gives $\phi(\sigma)=0$, and an energy defined as $U$ $=-\frac{9}{8} \sigma^{-3} \int_{\sigma}^{\infty} d r r^{2} \phi(r)$ to reproduce the usual parameters $\sigma$ and $\epsilon \equiv U_{\mathrm{LJ}}$ for the LJ model. Our effective $\phi_{\mathrm{Na}}$ description gives the values $\sigma_{\mathrm{Na}}=3.48 \AA$ and $U_{\mathrm{Na}}=0.1885 \mathrm{eV}$.

To clarify the relation between the shape of the potential and the melting temperature we have considered Eq. (1) as a 


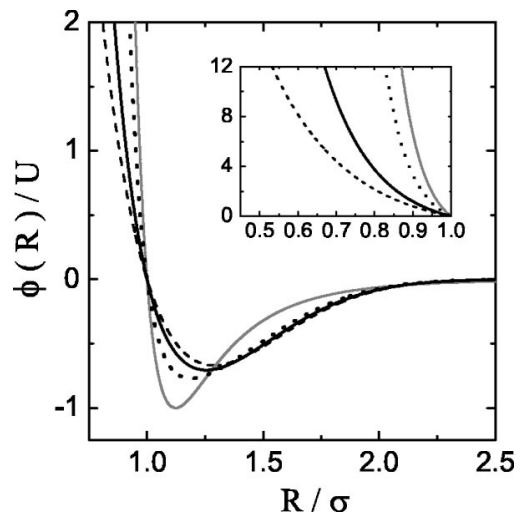

FIG. 1. Some of the effective pair potentials analyzed in this work. Reduced units $r / \sigma$ and $\phi / U$ are used with appropriate values for $\sigma$ and $U$ for each model potential, as defined in the text. Full line: Alkalilike potential $\phi_{\mathrm{Na}}$; thin full gray line: Lennard-Jones potential; dashed line: Potential with a softer repulsive part, $\phi_{\text {soft-1 }}$, and dotted line: Potential with a harder repulsive part, $\phi_{\text {repul }}$. Inset shows an enlarged view of the repulsive-part region.

variational family by modifying its parameters, thus obtaining new potentials, as follows. First, in order to study the role played by the repulsive part, we have analyzed the effect of its softness. By changing the value of $\alpha$ we have obtained two pair potentials, $\phi_{\text {soft-1 }}$ and $\phi_{\text {soft-2 }}$, with $\alpha=2 / 3 \alpha_{\mathrm{Na}}$ and $\alpha=3 / 4 \alpha_{\mathrm{Na}}$, respectively, which have a softer repulsive part than $\phi_{\mathrm{Na}}$, and a harder repulsive part potential $\phi_{\text {repul }}$, with $\alpha=2 \alpha_{\mathrm{Na}}$. In all these cases we have kept the $\phi_{\mathrm{Na}}$ values for $R_{1}$ and $\beta$, while $A_{0}$ and $A_{1}$ are changed as necessary in order to maintain the values of $\sigma$ and $U$. The values of the parameters for all potentials are given in Table I.

\section{The melting transition}

In this section we present an analysis of the phase diagrams associated with each of the alkalilike pair potentials defined previously. The phase diagrams include liquid-vapor and solid-vapor phase transitions. Three different techniques have been used to locate the corresponding phase boundaries: (i) NPT-MC simulations at zero pressure, (ii) direct simulation of a crystal slab and its melting to a liquid slab between vapor phases, and (iii) free-energy calculations for all the phases involved using NVT-MC simulations along with thermodynamic integration.

The first technique, based on NPT simulations, was used to obtain some information as to the approximate location of the melting temperature. A series of NPT-MC simulations at zero pressure and increasing, equidistant steps in temperature, were performed. 864 particles were used with periodic boundary conditions, and all potentials were truncated at $2.5 \sigma$. As input configurations for the simulations we used the structures that minimize the energy at $T=0$. The resulting equilibrium structures always have fcc symmetry, except for the $\phi_{\text {soft-2 }}$ potential which crystallizes with bcc symmetry. In all the NPT calculations to be presented below, the pressure is set to zero; this is obviously an approximation that is simply intended to provide an estimate for the melting temperature.

The empty symbols in Fig. 2 show the (averaged) equilibrium densities that result from the simulations at each tem-

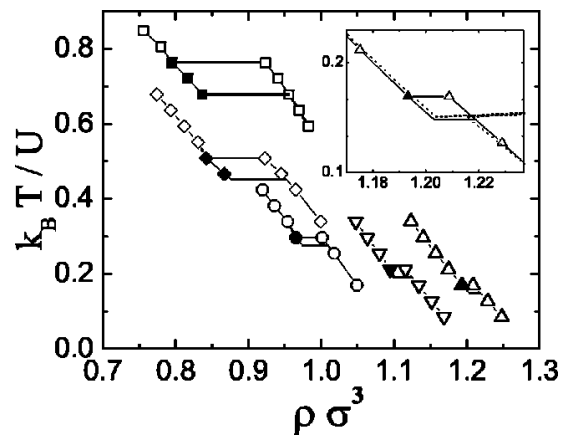

FIG. 2. The open symbols show the liquid-vapor and solid-vapor coexistence densities for different model potentials as obtained by NPT Monte Carlo simulations at zero pressure: $\phi_{\mathrm{Na}}$ (circles), $\phi_{\mathrm{LJ}}$ (squares), $\phi_{\text {repul }}$ (rhombus), $\phi_{\text {soft-1 }}$ (triangles up), and $\phi_{\text {soft-2 }}$ (triangles down). Closed symbols are results for the liquid density at coexistence with vapor from NVT Monte Carlo simulations initialized with a crystal slab. Inset shows an enlarged view close to the triple point for $\phi_{\text {soft- } 1}$ and includes results from thermodynamic integration (dashed line). Reduced temperatures $k_{B} T / U$ and densities $\rho \sigma^{3}$ are used with appropriate scaling constants $U$ and $\sigma$ for each model potential, as described in the text. Lines are guides to the eye.

perature. For each potential, the temperature at which a density jump is observed can be taken as an approximate location for the phase transition. Note that the location of the density gaps depends sensitively on the type of potential. In the following we will refer to the temperature at which melting occurs in the zero-pressure NPT simulations as the melting temperature, $T_{m}^{P=0}$.

The methodology based on the NPT simulations at zero pressure is only approximate; it amounts to assuming that the coexisting vapor is a perfect vacuum. As a consequence, it does not guarantee that one is obtaining states even close to the true thermodynamic states that coexist at equilibrium. Obviously the true coexistence pressure at the true triplepoint temperature, $T_{t}$, will be close to zero but finite, and the assumption $T_{t}=T_{m}^{P=0}$ will not exactly hold. The question of whether $T_{m}^{P=0}$ will overestimate $T_{t}$ or the other way round is a subtle one that we do not address here. In particular, issues such as system-size effects or, even more important, the presence of possibly persistent metastable states, are to be taken into account for a full discussion. Without further analysis all we can say is that, insofar as the coexistence vapor pressure is expected to be negligible and our system sizes are far from small, our estimate for $T_{t}$ will be relatively reasonable and, for most of the potentials explored in this study, we believe that this is indeed the case. One exception may be the LJ potential which, compared to the other potentials, presents a non-negligible vapor pressure at coexistence.

To remedy this shortcoming, we used a second technique to locate the triple point and the different phase boundaries which involves the direct simulation of the liquid-vapor interface, from which bulk liquid and vapor (coexistence) densities can be extracted. Of course, from these simulations one can obtain, as a bonus, the properties of the interface itself; these results will be discussed in Sec. III. A series of NVT-MC simulations were carried out, with 2592 particles in a rectangular box, periodically replicated in all three directions. Particles were arranged in slab geometry by dividing the central box into nine cubes of equal size along the $z$ 
TABLE II. Critical temperature $T_{c}$, triple-point temperature $T_{t}$, and relative volume change on melting $\Delta V / V$ for several effective pair potentials. $T_{m}^{P=0}$ is the triple point temperature calculated using the criterion of our previous work (Ref. 13), whereas $T_{t}$ corresponds to the values obtained using the criterion proposed in this work. Reduced temperatures $k_{B} T / U$ are used.

\begin{tabular}{lccccc}
\hline \hline Potential & $T_{c}$ & $T_{m}^{P=0}$ & $T_{t}$ & $T_{t} / T_{c}$ & $\Delta V / V$ \\
\hline soft-1 & 1.43 & 0.1696 & $0.15 \pm 0.02$ & 0.10 & $0.6 \%$ \\
soft-2 & 1.35 & 0.2120 & $0.19 \pm 0.02$ & 0.14 & $1.2 \%$ \\
Na & 1.25 & 0.2968 & $0.27 \pm 0.02$ & 0.22 & $3.6 \%$ \\
repul & 1.22 & 0.5088 & $0.45 \pm 0.02$ & 0.37 & $9.0 \%$ \\
LJ & 1.21 & 0.7632 & $0.68 \pm 0.02$ & 0.56 & $14.8 \%$ \\
Hg & 1.17 & 0.3392 & $0.32 \pm 0.02$ & 0.27 & $14.5 \%$ \\
\hline \hline
\end{tabular}

axis. The three inner cubes were filled with particles, the remaining six boxes being empty which creates two similar, flat interfaces in the system. For temperatures below the critical temperature, $T_{c}$, the systems equilibrate to give a slab of liquid, with vapor on both sides. Our typical simulation sizes are $L_{x}=L_{y}=8.5-10.5 \sigma$ for the transverse size and $L_{z}$ $=76.5-95 \sigma$ for the longitudinal size, and sampling times in excess of $3 \times 10^{5} \mathrm{MC}$ steps were necessary to achieve equilibration.

Filled symbols in Fig. 2 show the resulting averaged densities obtained from the slab simulations using crystalline initial configurations. We only present those results that do not coincide with the corresponding NPT (zero-pressure) results. As can be seen, the slab simulation states melt at a lower temperature. This difference is larger, in absolute temperature, for the systems with higher melting temperatures, $\phi_{\mathrm{LJ}}$ and $\phi_{\text {repul }}$, although the difference relative to $T_{t}$ are similar.

In the simulations presented in our previous letter ${ }^{13}$ we were careful enough to insure that the slab was in a liquid configuration, so we adopted the conservative criterion of defining an approximate melting temperature as the higher temperature for which the NPT simulations at zero pressure predicted a solid configuration and at the same time the NVT-slab simulations still predicted a liquid bulk phase. These values, shown in Table II as $T_{m}^{P=0}$, are indicated in Fig. 2 by the upper horizontal solid line for each potential. However, it is known that in the case of the LJ potential the true melting temperature occurs at a temperature very close to that at which the slab is melting in the NVT-slab simulation. Based on this finding, the melting of the slab would in principle be a better criterion, save for the presence of finitesize effects. As the correct determination of the triple-point temperature $T_{t}$ is very important to interpret our interfacial simulations, it would be desirable to have an alternative criterion to establish the location of the liquid-solid transition more rigorously, especially in the case of the soft-core potentials, whose melting properties are not so well known.

\section{Thermodynamic integration for $\phi_{\text {soft-1 }}$}

We have performed independent simulations with a view to accurately locate the triple point by rigorously applying the thermodynamic equilibrium conditions, namely, the equality of pressures, temperatures and chemical potentials of the phases involved. Our aim is the determination of coexistence parameters from calculations of the free energies, which requires performing thermodynamic integration of the pressure and the energy, two mechanical quantities that can be directly measured by simulation. Since these simulations are relatively demanding, and given that hard repulsive potentials, such as the LJ potential, have been investigated in much more detail, we have focused on the pair potential $\phi_{\text {soft-1 }}$ to obtain, at least in one case, the true triple point and to assess the validity of the zero-pressure assumption in the NPT simulations directly. The Helmholtz free energies of vapor, liquid and solid phases were computed and the relative phase stability obtained; coexistence parameters can then be extracted by applying a standard Maxwell construction.

Depending on the phase the calculation involves a different technique. For a whole range of temperatures around the triple point the free energy of the vapor phase can be simply and accurately obtained from a two- or three-term virial expansion, since the vapor density is expected to be very low. The free energy of the liquid is calculated by thermodynamic integration by devising a reversible path split in two parts: First, we integrate along a supercritical isotherm from a region close to the ideal gas up to a density over the (expected) density of the triple point. Then, we perform thermodynamic integration in temperature along an isocore down to a temperature below the (expected) triple point temperature. The free-energy contribution from the first trajectory (the supercritical isotherm) is evaluated by integrating the compressibility factor, which is obtained from the radial distribution function using NVT-MC simulations. The contribution from the second trajectory (the isocore) is obtained from integration of the internal energy, also obtained by NVT MC simulations. In all cases the integrands are smooth functions of the corresponding variables and thus amenable to polynomial fitting which leads to analytical expressions for the free energy. Finally, to obtain the free energy of the solid, we have used the coupling parameter technique ${ }^{18}$ starting from of an Einstein crystal, whose free energy is known exactly. Simulations were done in the NVT ensemble with $N=256$ particles. All simulations were equilibrated with $4 \times 10^{4} \mathrm{MC}$ steps and the radial distribution function and the energy averaged over the next $4 \times 10^{4} \mathrm{MC}$ steps. Virial coefficients for the vapor phase were calculated directly in terms of cluster integrals using numerical quadrature.

In the inset of Fig. 2 a detailed comparison of the results from thermodynamic integration with the zero-pressure NPT and NVT-slab simulations in the region around the triple- 


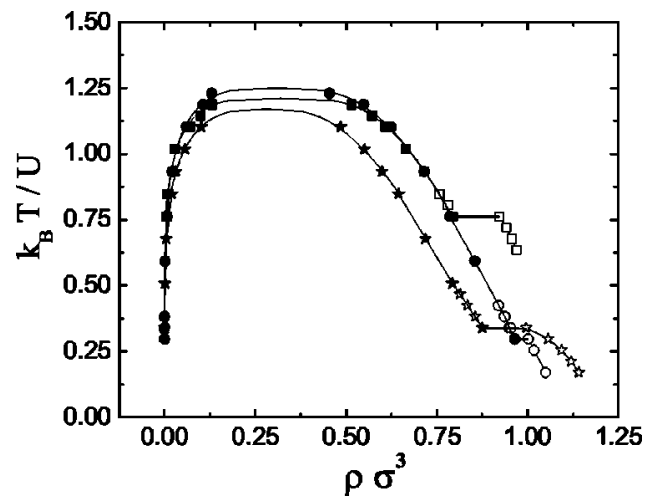

FIG. 3. Comparison of global phase diagrams obtained for the model potentials $\phi_{\mathrm{Na}}$ (circles), $\phi_{\mathrm{LJ}}$ (squares) and $\phi_{\mathrm{Hg}}$ (stars). Filled symbols are results for the liquid-vapor co-existence densities from NVT Monte Carlo simulations in slab geometry. Open symbols correspond to NPT Monte Carlo results at zero pressure shown in Fig. 2. Reduced temperatures $k_{B} T / U$ and densities $\rho \sigma^{3}$ are used and the lines are guides to the eye.

point is made. Clearly, the melting temperature obtained by thermodynamic integration, $k_{B} T_{t} / U=0.15$, is remarkably similar to that obtained from the NVT-slab simulations, showing once more that the slab simulations provide a better criterion to establish a melting temperature than isobaricensemble simulations. Therefore, we have established a new criterion to define a melting temperature by taking the average of the highest temperature for which the slab is solid and the lowest temperature for which the slab is liquid; this temperature is referred to in Table II as $T_{t}$. In the case of the $\mathrm{LJ}$ potential, we have preferred to define $T_{t}$ as the lowest temperature for which the slab is "liquid" since for this temperature we have been lucky enough to observe direct coexistence of vapor, liquid and solid phases for one of the temperatures selected in the simulations. The melting temperatures according to the new definition are indicated in Fig. 2 for each potential by means of the lower horizontal solid line; the corresponding values are given in Table II. As can be seen from the table, the change of definition for the melting temperature is not very significant. For the sake of comparison, the table also contains our estimates for $T_{m}^{P=0}$ from our previous letter. ${ }^{13}$

\section{The liquid-vapor phase diagram}

The NVT-slab simulations directly provide coexistence densities for the liquid and vapor phases at each temperature above the melting point. The resulting phase diagrams in the temperature-density plane are depicted in Figs. 3 and 4, where coexistence densities for the liquid-vapor (filled symbols) and also for solid-vapor transitions (open symbols), each in the appropriate temperature range, are represented. Fitting the high-temperature liquid-vapor coexistence densities to a form $\rho_{l}-\rho_{v} \propto\left(T-T_{c}\right)^{\beta}$ provides an estimate for the critical temperature $T_{c}$ for each potential. These values are given in Table II. The values for $\beta$ resulting from the fit, $\beta$ $=0.327$, are compatible with the established value.

An important conclusion to be drawn from the results of Figs. 3 and 4 is that all potentials have numerically similar reduced critical temperatures $k_{B} T_{c} / U$, with only the softrepulsive potentials having an appreciable effect over the

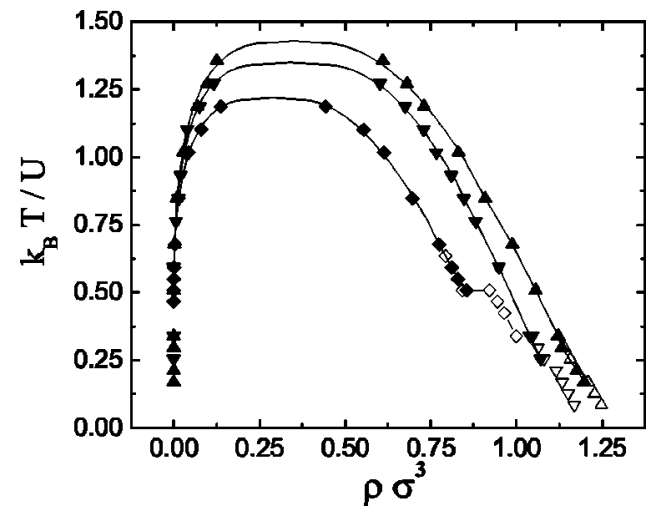

FIG. 4. As in Fig. 3 but for the potentials $\phi_{\text {soft- } 1}$ (triangles up), $\phi_{\text {soft-2 }}$ (triangles down) and $\phi_{\text {repul }}$ (rombhus).

scaled critical temperature (an increase of $12 \%$ for $\phi_{\text {soft-1 }}$ and $8 \%$ for the $\phi_{\text {soft-2 }}$ potential). Note that the critical temperature obtained with the pair potential $\phi_{\mathrm{Na}}(r)$ is $T_{c}$ $\approx 1.22 U_{\mathrm{Na}} / k_{\mathrm{B}}=2669 \mathrm{~K}$, i.e., less than $8 \%$ above the experimental value for $\phi_{\mathrm{Na}}(2483 \mathrm{~K})$. This agreement is quite satisfactory, considering the simplicity of our representation and the limited amount of information used to extract this effective pair potential.

By contrast, a remarkable feature of the phase diagrams of Figs. 3 and 4 is the gross differences in the ratio $T_{t} / T_{c}$ for the different model potentials, which amounts to about 0.66 for the LJ potential, 0.24 for $\phi_{\mathrm{Na}}$ and 0.13 for $\phi_{\mathrm{soft}-1}$. The value for $\phi_{\mathrm{Na}}$ is still high compared with the experimental results for the alkali metals, reflecting the limited accuracy of our simple pair-potential model to incorporate the subtle many-body effects in real metals, but the qualitative effect of a low reduced melting temperature may certainly be obtained with these simple pair potentials. In fact, using the artificially softened version $\phi_{\text {soft-1 }}$ (see Fig. 1) we obtain a small increase of $T_{c}$ but a much larger drop of $T_{t}$, resulting in a ratio $T_{t} / T_{c} \approx 0.13$ which is very similar to the experimental value for Hg. Similarly $\phi_{\text {soft-2 }}$ has $T_{t} / T_{c} \approx 0.15$ which is very close to the experimental values for alkali metals. Although it is difficult a priori to single out the features that a pair potential should possess in order to give a low value for the ratio $T_{t} / T_{c}$, the use of a simple effective description and the artificial enhancement of some of its features may help in establishing the relevance of these features. Thus, it seems that if the repulsive part is softened, then $T_{t}$ decreases, while if the repulsive part is hardened, the melting temperature increases; for instance, the ratio $T_{t} / T_{c}$ increases to 0.40 for $\phi_{\text {repul }}$, to be compared with 0.22 for $\phi_{\mathrm{Na}}$. By contrast, for the alkalilike potentials, the range of the attractive part has a negligible effect on $T_{t} / T_{c}$, as we have verified by studying a shorter-ranged potential with $\beta=2 \beta_{\mathrm{Na}}$. For this potential we obtain a ratio $T_{t} / T_{c}=0.27$, which is comparable to that for the $\phi_{\mathrm{Na}}$ potential. Similar results are obtained if the range of the attractive part is increased. In the light of these results we can conclude that for alkalilike potentials the decrease in melting temperature can be linked to an increasing softness of the repulsive part of the pair-potential interaction.

Finally, Fig. 5 shows the comparison between the NVTslab simulations and the direct free-energy calculations based 


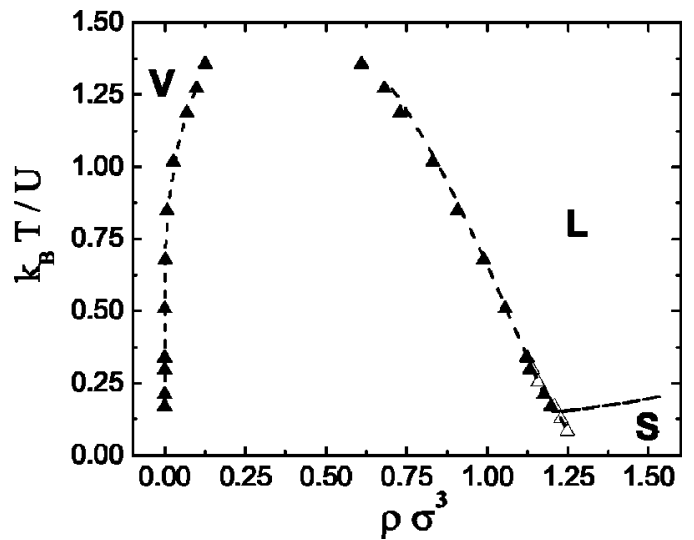

FIG. 5. Global phase diagram for model potential $\phi_{\text {soft-1 }}$, as obtained using thermodynamic integration (dashed line), NVT Monte Carlo simulations in slab geometry (filled up-triangles), and NPT Monte Carlo at zero pressure (open up-triangles).

on thermodynamic integration for the case of the soft-1 potential. The agreement is quite satisfactory, the only (minor) differences arising in the high-temperature region where the interfacial calculations predict a slightly lower liquid density.

\section{B. The mercurylike systems}

\section{Pair potential and phase diagram}

By analyzing the alkalilike pair potentials we have found that the presence of a soft repulsive part in the interparticle pair potential tends to lower the triple point temperature, a situation that will later be seen to be associated with the presence of nonmonotonic liquid-vapor density profiles for temperatures close to the triple point. However, the question arises as to whether other pair potentials with different functional forms and, in particular, different radial dependence in the short-range repulsive part, could also generate a low triple point temperature. In fact, it is known that the radial distribution function of $\mathrm{Hg}$ (with both a low melting temperature and surface oscillations in the free liquid surface) exhibits a much steeper radial dependence at short distances near the melting point than $\mathrm{Cs}$ and $\mathrm{Rb}$, and this reflects the hard-repulsive nature of the effective pair potential of metallic $\mathrm{Hg}$ that contrasts with the softer repulsive part of the effective potentials for liquid alkali metals. ${ }^{17}$ Fitting the experimental isochoric thermal pressure coefficients to those based on Monte Carlo calculations using smooth inverse power-law interparticle potentials $1 / r^{n}$, the best-fit values for $n$ are obtained in the interval 15 to 16 for densities close to the melting point, which indicates that the repulsive part of $\mathrm{Hg}$ is harder than that of the LJ potential $(n=12)^{17}$ and, indirectly, that the repulsive pair-potential softness (or rather its lack thereof) cannot be an explanation for the low melting temperature of $\mathrm{Hg}$.

To investigate this point we have analyzed the effective pair potential for mercury, $\phi_{\mathrm{Hg}}(r)$, obtained from the inverse-method result ${ }^{19}$ for the structure factor at a hot metallic state $\left(T=1273 \mathrm{~K}\right.$, and density $\left.\rho=10.98 \mathrm{~g} / \mathrm{cm}^{3}\right)$. The potential is presented in Fig. 1 in reduced units, with $\sigma_{H g}$ $=2.91 \AA$ and $U_{\mathrm{Hg}}=0.12633 \mathrm{eV}$. As we can see the shape of $\phi_{\mathrm{Hg}}(r)$ is compatible with evidence obtained from the ex-

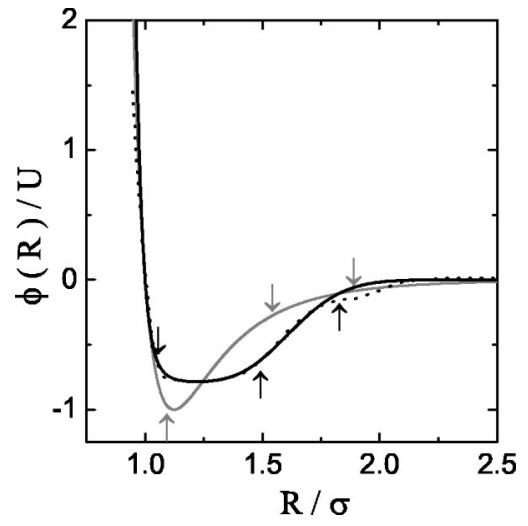

FIG. 6. Effective pair potentials for $\mathrm{Hg}$. Dotted line: Potential derived from the experimental structure factor $\left(T=1273 \mathrm{~K}\right.$ and $\left.\rho=10.98 \mathrm{~g} / \mathrm{cm}^{3}\right)$ by the inverse method; full line: Effective model potential for $\mathrm{Hg}, \phi_{\mathrm{Hg}}$; full gray line: Lennard-Jones potential. The arrows indicate the position of the first, second and third nearest neighbor for the fcc solid at $T=0$. Black arrows: $\phi_{\mathrm{Hg}}$; gray arrows: LJ potential.

perimental structure factor: The attractive well is broader than in the LJ and alkali potentials, whereas the repulsive part is harder. For technical reasons we have preferred not to use this potential directly but the following fit:

$$
\begin{aligned}
& \phi_{\mathrm{Hg}}(r)=A_{0} e^{-\alpha r}-A_{1}\left(e^{-\beta\left(r-R_{1}\right)^{2}}+e^{-\beta\left(r-R_{2}\right)^{2}}\right), \\
& \text { with } \quad A_{0}=2.06162 \times 10^{14} \mathrm{eV}, \quad \alpha=12.0237 \AA^{-1}, \quad A_{1} \\
& =0.07537 \mathrm{eV}, \quad \beta=1.2265 \AA^{-2}, \quad R_{1}=2.9527 \AA, \quad \text { and } R_{2} \\
& =4.1233 .
\end{aligned}
$$

Carrying out the same procedure used with the alkalilike potentials (i.e., interfacial NVT simulations and calculation of bulk coexistence densities) the critical temperature for the effective pair potential $\phi_{\mathrm{Hg}}(r)$ can be obtained at $T_{c}$ $\approx 1.15 U_{\mathrm{Hg}} / k_{\mathrm{B}}=1686 \mathrm{~K}$, which is within $5 \%$ of the experimental value for $\mathrm{Hg}(1751 \mathrm{~K})$. As we can be seen in Table II the ratio $T_{t} / T_{c}$ in this case is $\sim 0.27$, which is close to that for $\phi_{\mathrm{Na}}$ but much lower than the value obtained for $\phi_{\mathrm{LJ}}$. Note that, although the repulsive part of $\phi_{\mathrm{Hg}}$ is harder than that of $\phi_{\mathrm{LJ}}$, the former has a relatively low value of $T_{t} / T_{c}$ which indicates that features other than the softness of the repulsive part may be relevant to generate a low triple-point temperature.

The most obvious difference between $\phi_{\mathrm{Hg}}$ and $\phi_{\mathrm{LJ}}$ in Fig. 6, is the broad and flat well potential of $\phi_{\mathrm{Hg}}$ which takes similar values at the location of first and second nearest neighbors in the fcc crystal, while the third-neighbor shell is nearly outside of the well. This fact may explain the low value of $T_{t}$, since the second-neighbor shell contains only 6 atoms, while the third has 24 . The disordered packing of liquid configurations may then be more efficient than the crystalline compact packing structures to accumulate neighbors within the range of the well potential.

\section{The square-well system}

To explore this hypothesis we have studied the melting temperature of square-well fluids of variable width as a function of $\lambda$, the ratio between the range of the potential well and the hard-sphere diameter. This potential has been widely studied for $\lambda \approx 1$ since these values are believed to be rel- 
evant to model effective interparticle interactions in colloidal fluids. The range $1.3<\lambda<1.8$ has been extensively studied but only as far the liquid-vapor transition and critical properties $^{20}$ are concerned. The fluid-solid transition and properties close to the melting point are less well understood. To our knowledge the only analysis is that due to Young and Alder $^{21}$ who mapped out the entire phase diagram for the case $\lambda=1.5$ using a combination of second-order perturbation theory and molecular-dynamics simulations. Their results show a very complex phase diagram, with several solid-solid transitions and reentrant behaviors. This structure, however, may be very sensitive to the approximate nature of their theory since it relies on very subtle differences in free energies. In any case a systematic study using numerical simulation for a variety of values of $\lambda$ is an extremely laborious task outside the scope of the present paper.

In an effort to elucidate the origin of the decrease in triple-point temperature associated with a relatively wide and flat attractive potential we have formulated a linear perturbation theory and analyzed the liquid-solid phase behavior as a function of the range parameter $\lambda$ in a wide interval. We stress that we are only interested in trying to find trends and indications for the decrease in melting temperature rather than a detailed study of the stability of the many phases that might appear in this system. As mentioned in the introduction, perturbation theories perform better in the case of hardcore systems than for systems having a soft core, though the presence of strong correlations in the liquid together with the necessity to explore the low temperature region may decrease the accuracy of the results of perturbation theory. Despite this, we believe that the linear perturbation theory may at least indicate stability trends for the solid as the squarewell range $\lambda$ is varied. The theory approximates the Helmholtz free-energy of the system (either liquid or solid) by

$$
\mathcal{F}[\rho]=\mathcal{F}_{h s}[\rho]+\frac{1}{2} \iint d \mathbf{r} d \mathbf{r}^{\prime} \phi\left(\left|\mathbf{r}-\mathbf{r}^{\prime}\right|\right) \rho^{(2)}\left(\mathbf{r}, \mathbf{r}^{\prime}\right),
$$

where $\mathcal{F}_{h s}[\rho]$ is a density functional for the hard-sphere reference system, and $\rho^{(2)}\left(\mathbf{r}, \mathbf{r}^{\prime}\right)$ is the pair distribution function of the hard-sphere system. For the liquid phase $\rho^{(2)}\left(\mathbf{r}, \mathbf{r}^{\prime}\right)$ $=\rho^{2} g\left(\left|\mathbf{r}-\mathbf{r}^{\prime}\right|\right)$, in terms of the radial distribution function $g\left(\left|\mathbf{r}-\mathbf{r}^{\prime}\right|\right)$, which we approximate by the very accurate representation due to Verlet and Weis; whereas for the solid phase we simply approximate $\rho^{(2)}\left(\mathbf{r}, \mathbf{r}^{\prime}\right)=\rho(\mathbf{r}) \rho\left(\mathbf{r}^{\prime}\right) \Theta(\mid \mathbf{r}$ $\left.-\mathbf{r}^{\prime} \mid\right)$, with the Heaviside step function to exclude the overlap of the hard-sphere cores. The hard-sphere contribution $\mathcal{F}_{h s}[\rho]$ for the liquid is described using the quasiexact freeenergy expression by Carnahan and Starling. In the the solid we have used the free energy DF obtained by Tarazona ${ }^{22}$ and based on fundamental measure theory, which improves previous DF approximations since it includes the unit cell anisotropy of the fcc lattice and the description of the metastable bcc lattice. The results of this theory are contained in Fig. 7 where the triple-point temperature, suitably scaled by the critical temperature, is represented as a function of the inverse range parameter $\lambda^{-1}$. Filled symbols outside the shaded vertical band indicate that for the corresponding values of $\lambda$ there is coexistence between a vapor, a liquid and a fcc solid at a temperature indicated by the symbol. In this

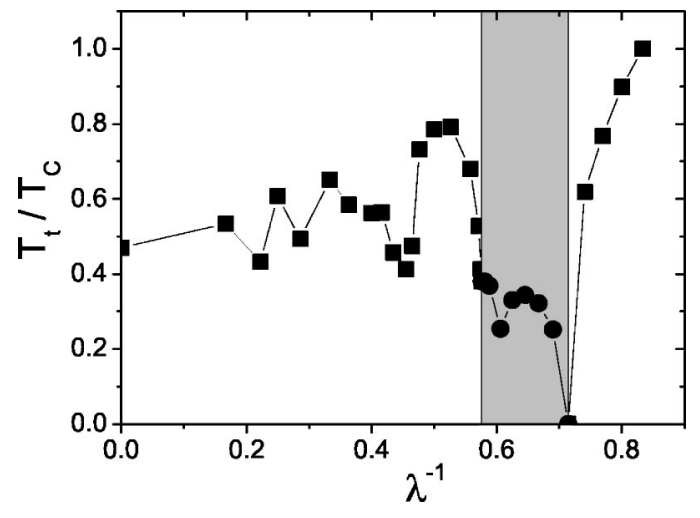

FIG. 7. Ratio of triple-point $T_{t}$ to critical $T_{c}$ temperatures for a square-well potential as a function of the variable well width. $\lambda$ is the range of the potential well in hard-sphere diameter units. The squares indicate a stable fcc solid structure whereas circles correspond to situations where the fcc structure is unstable with respect other solid structures or the liquid phase. The interval in $\lambda$ where the latter situation occurs has been shaded.

region the triple-point temperature relative to the critical temperature is relatively high. The shaded band represents an interval in $\lambda$, defined approximately by $1.75<\lambda<1.4$, where the solid compact structures fcc and hcp become unstable with respect to the liquid, other crystalline structures dominating the phase behavior which, in any case, is extremely complex. The case $\lambda=1.4\left(\lambda^{-1}=0.71\right)$ is particularly interesting since the fcc, bcc, hcp, and rhombohedral lattice structures are less stable than the liquid down to zero temperature so that the triple-point temperature corresponding to the more stable phase appears to be very low. Obviously the limited accuracy of perturbation theory does not guarantee the quantitative nature of these findings; however, we believe that the main conclusion, namely the destabilization of the solid compact structures in the range $1.75<\lambda<1.4$ is a qualitatively correct effect. As a test of our linear perturbative theory we can compare our results with the second-order results obtained by Young and Alder ${ }^{21}$ for $\lambda=1.5$. Their estimate $T_{t} / T_{c}=0.35$, even lower that the obtained by us (0.46), confirms our conclusion that in the interval $1.75<\lambda$ $<1.4$ the triple-point temperature must be lower than outside the interval.

The lowering of this temperature is associated to an instability of the crystalline phases (either compact or noncompact) with respect to the liquid, and can be rationalized as follows. For wide enough square wells the first correlation shell will be contained entirely inside the well. This gives a (practically) constant contribution to the free energy. However, the distance of the second correlation shell to a central particle is close to the values of $\lambda$ that we are considering and, as a result, part of this shell actually lies inside the well. Thus, the variation of the free energy with density comes mainly from this second correlation shell and it may occur that, at a given fixed density, the system finds it more favorable to become a liquid by spreading out the corresponding second-shell correlation distribution in order to lower the attractive energy with little entropy cost, thus lowering the free energy. This mechanism might be somewhat weakened in a more complete calculation that included some relaxation of the crystalline correlation structure (note that our first- 


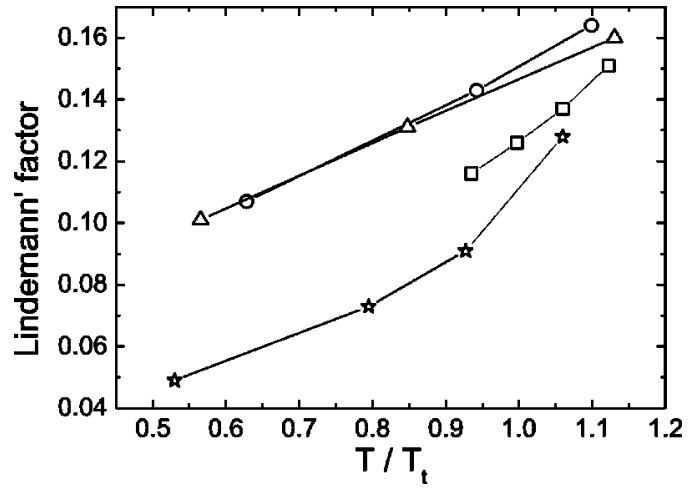

FIG. 8. Lindemann parameter as a function of temperature, for temperatures close to the triple-point temperature $T_{t}$ and along the zero-pressure line. Circles: $\phi_{\mathrm{Na}}$; triangles: $\phi_{\text {soft-1 }}$; squares: $\phi_{\mathrm{LJ}}$, and stars: $\phi_{\mathrm{Hg}}$. The lines are only intended as guides to the eye.

order perturbation theory uses a simple step-function for the radial distribution function, so it implicitly assumes a rigid correlation structure).

These results obtained for the square-well potential confirm the mechanism responsible for the associated low triplepoint temperature in $\phi_{\mathrm{Hg}}$. The width of the attractive well of $\phi_{\mathrm{Hg}}$ is similar to that of a square well with $\lambda \approx 1.5$; this, in turn, implies that its solid compact structures will tend to be less stable, with noncompact structures such as bcc or rhombohedral appearing as the more stable solid phases, which makes the triple-point temperature to decrease. This conclusion is consistent with the well-known result that Hg crystallizes with a rhombohedral crystal structure.

\section{General remarks}

In the previous sections we have presented two different families of effective pair potential that have low melting temperatures. The first family includes potentials with a soft repulsive part, whereas in the second the potential contains a broad and flat attractive well with a width that destabilizes solid compact structures. An interesting question is whether or not melting in these systems proceeds differently from melting in LJ-like systems. One way to analyze possible differences is to study the applicability to our systems of two popular, semiempirical criteria for localizing the melting transition: The Hansen-Verlet and the Lindemann criteria.

The Lindemann criterion ${ }^{23,24}$ applies to the solid and postulates that the crystal melts when the mean-square amplitude of vibration of the particles about their mean position, relative to the crystal nearest-neighbor distance, reaches a critical value that has been shown ${ }^{24}$ to vary between 0.095 and 0.16. We have evaluated the Lindemann parameter by averaging the root-mean-square displacement of each particle from its reference lattice site, which in the simulations is shifted with the center of mass of the system. As can be seen in Fig. 8, the Lindemann parameter at the melting temperature for the potentials that we have analyzed varies in the range $0.11-0.15$. Rather than the absolute value itself, it is more interesting to notice that the temperature dependence of the parameter is different from one model potential to another. More specifically, for the soft-repulsive potentials

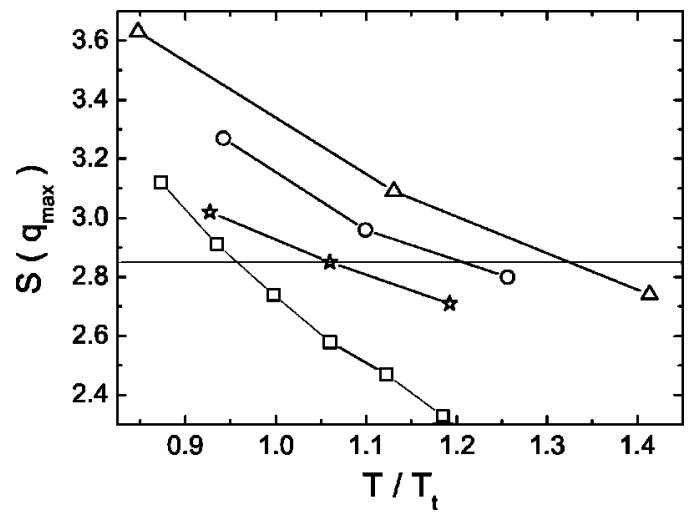

FIG. 9. Amplitude of the first peak of the structure factor $S\left(q_{\max }\right)$ as a function of temperature, for temperatures close to the triple-point temperature $T_{t}$ and along the zero-pressure line. Circles, $\phi_{\mathrm{Na}}$; triangles, $\phi_{\text {soft- } 1}$; squares, $\phi_{\mathrm{LJ}}$, and stars, $\phi_{\mathrm{Hg}}$. The lines are only intended as guides to the eye.

$\phi_{\text {soft- } 1}$ and $\phi_{\mathrm{Na}}$, the dependence is rather weak and linear, whereas for $\phi_{\mathrm{Hg}}$ the Lindemann parameter has a low value which, as the melting temperature is approached, increases quite dramatically. These two different behaviors indicate rather different melting mechanisms at the molecular level. In any case, the larger values of the Lindemann parameter for the soft-repulsive potentials and its weaker temperature dependence are clearly a consequence of the fact that the effective potential felt by a molecule is very flat, which allows the molecule to vibrate with a larger amplitude. In the case of the $\mathrm{Hg}$ potential, molecules are more constrained in their cage due to the abrupt nature of the potential and its particular width, which hinders molecular vibrations; only close to the melting temperature are molecules more free to vibrate and eventually escape from their cage.

The Hansen-Verlet criterion, ${ }^{25,24}$ which applies to the liquid, states that the amplitude of the first peak of the structure factor reaches the universal value $S\left(q_{\max }\right)=2.85 \pm 0.1$ at melting. This value has been tested for Lennard-Jones and hard-sphere potentials. ${ }^{24}$ However, for inverse-power potentials, Agrawal and $\mathrm{Kofke}^{26}$ found that, in some cases, $S\left(q_{\max }\right)$ takes on values approaching 3.2 . For the model potentials analyzed in this work, we have calculated the structure factor by numerical Fourier transform of the radial distribution function. The latter has been calculated by NVT Monte Carlo simulation with $\mathrm{N}=1372$ particles and extended analytically by fitting the tail of the simulation result to the asymptotic form

$$
g(r)=1+A \frac{e^{-\alpha\left(r-r_{o}\right)}}{r} \cos q\left(r-r_{o}\right)+\cdots .
$$

In Fig. 9 we show $S\left(q_{\max }\right)$ at temperatures around $T_{t}$ for various pair potentials. As can be seen the potentials with a harder repulsive part, $\phi_{\mathrm{Hg}}$ and $\phi_{\mathrm{LJ}}$, satisfy the HansenVerlet criterion reasonably and exhibit a $S\left(q_{\max }\right)$ value close to 2.85. However the potentials with a softer repulsive part do not seem to follow the criterion, showing systematically higher values, $S\left(q_{\max }\right) \approx 3.15$ for $\phi_{\mathrm{Na}}$ and $\approx 3.35$ for $\phi_{\text {soft- } 1}$. These results are in agreement with the Agrawal and Kofke ${ }^{26}$ results for the inverse-power potentials $1 / r^{n}$ and diminish the 


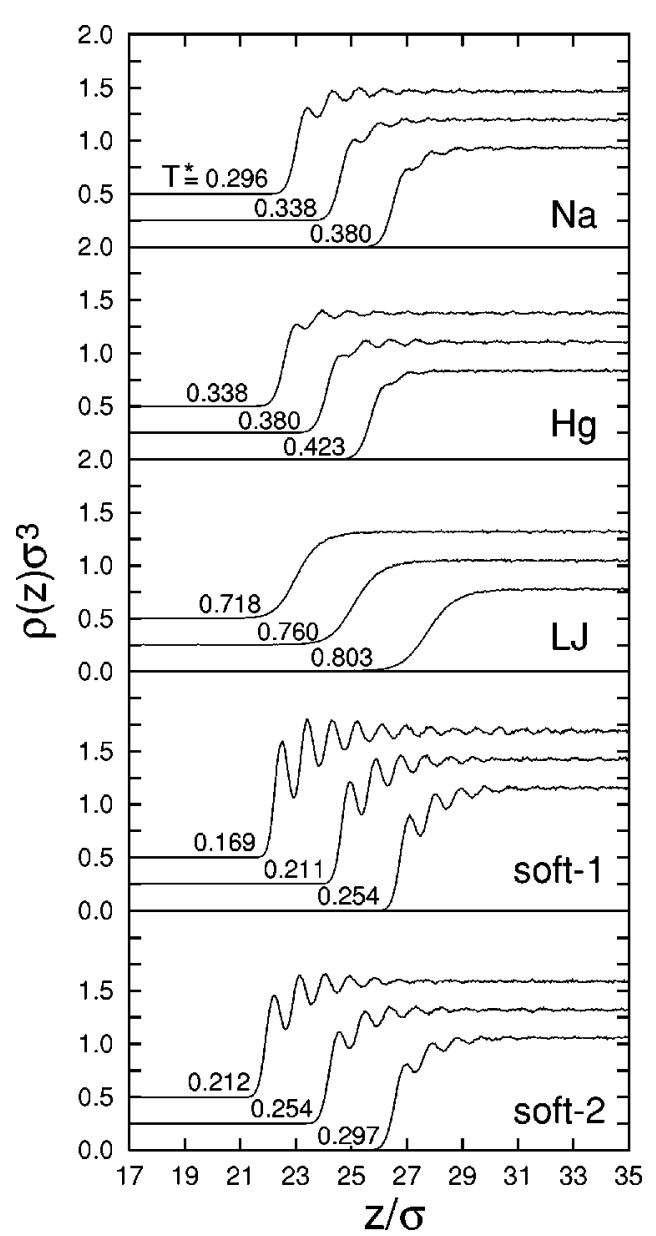

FIG. 10. Liquid-vapor density profiles in reduced units, $z / \sigma$ and $\rho(z) \sigma^{3}$ for model potentials $\phi_{\mathrm{Na}} \phi_{\mathrm{Hg}}, \phi_{\mathrm{LJ}}, \phi_{\mathrm{soft}-1}$, and $\phi_{\mathrm{soft}-2}$. The results in each case are for three values of $T^{*}=k_{B} T / U$ just above the melting point.

support for the generally accepted consensus that the Hansen-Verlet rule is quite universal. One could think that an uncertainty in the estimate for the triple-point temperature may be the origin of this discrepancy. However, at least for the case of the $\phi_{\text {soft-1 }}$ potential, our thermodynamicintegration results indicate that the triple-point temperature has been correctly estimated.

Finally, we have studied the relative volume change on melting, which turns out to vary strongly from one potential to the other. From Table II one can observe the general trend that the relative volume change decreases quite substantially as the repulsive part of the potential becomes softer.

\section{THE LIQUID-VAPOR DENSITY PROFILE}

Results for the liquid-vapor surface structure, shown in Fig. 10, were obtained from the same NVT-MC simulations (in slab geometry) used to obtain the liquid-vapor coexistence curve. The reported liquid-vapor density profiles are seen to be independent of the initial configuration of the slab (either liquid or solid), which provides a further check on equilibration but, in the NVT-slab simulations, melting is consistently observed at slightly lower temperatures than in the NPT simulations. Density profiles are presented at three different temperatures for each model pair-potential. The lowest temperature is always that for which the crystal slab is first observed to melt (but the crystal in the NPT simulation may have not melted), the next profile corresponding to the first temperature for which bulk melting is observed in the NPT bulk simulations, while the third profile corresponds to a higher temperature.

As can be seen in Fig. 10, the $\phi_{\mathrm{Na}}$ and $\phi_{\mathrm{Hg}}$ potentials lead to profiles with weak oscillatory structures, damped as the temperature increases. The soft-repulsive model potentials $\phi_{\text {soft- } 1}$ and $\phi_{\text {soft- } 2}$ (with $T_{t} / T_{c}$ ratios similar to the experimental values for metals) give profiles with a substantial amount of surface layering structure at $T_{t}$. However, the density profiles for the LJ model have the usual monotonic behavior observed in many previous works and the same behavior is observed for the density profiles with $\phi_{\text {repul }}$ (which gives a ratio $T_{t} / T_{c}$ higher than $\phi_{\mathrm{Na}}$ but lower that $\left.\phi_{\mathrm{LJ}}\right)$. Our detailed study of the phase diagrams indicates that the oscillatory structures are not artifacts of having overcooled metastable liquids and it confirms that quite different pair interaction model potentials give similar amplitudes of surface layering at similar reduced temperatures. This seems to indicate that surface layering is a generic property of cold liquid surfaces, which appears whenever $T_{t} / T_{c}$ is low enough to stabilize the liquid at temperatures well below $T_{c}$, irrespective of the physical mechanism responsible for the low $T_{t}$.

Within the family generated by $\phi_{\mathrm{Na}}$ and its arbitrarily softer and harder core variations the trend of $T_{t}$, shown in Table II, makes clear the role of the soft cores in lowering $T_{t}$ and closing the gap between liquid and solid densities at the triple point, while the density profiles for different models at the same reduced temperature are very similar. The $\phi_{\mathrm{Hg}}$ potential has a completely different mechanism to lower $T_{t}$, and most properties analyzed in the previous section put it on the opposite side of the soft core models, with respect to the prototypic LJ potential. However, the density profiles are again similar that those of the $\phi_{\mathrm{Na}}$. We have also obtained surface layering for the square-well liquid with $\lambda=1.72$ at $k_{B} T / U=0.38$ and 0.423 . This potential represents an enhanced version of the $\phi_{\mathrm{Hg}}$ potential with respect to the proposed mechanism to lower $T_{t}$. Again, the corresponding density profiles are similar to those of the other model potentials. However, these results are not presented in this paper since we have been unable to confirm whether or not the bulk liquid phase is stable for the above thermodynamic conditions. Moreover, by cooling the liquid slabs corresponding to the LJ and hard-repulsive potentials well below $T_{t}$, we have also observed similar oscillatory structures, but the interpretation of such MC-metastable states is subtle and they indeed may be impossible to observe experimentally.

In a recent work, ${ }^{14}$ we have analyzed the relationship between the oscillatory structures of the density profiles and those of the radial distribution functions for the bulk liquids, along with the density-functional predictions for the FisherWidom line. Also in that work we studied the temperature dependence of the layering amplitude for the $\phi_{\text {soft- } 1}$ potential and found that the leading contribution for the damping of the structure as $T$ increases comes from the capillary waves (CW) broadening of the liquid-vapor interface, which im- 


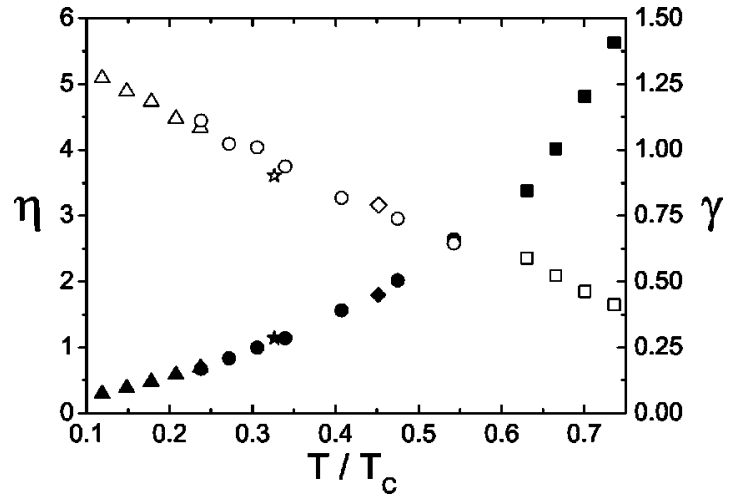

FIG. 11. The capillary-wave exponent $\eta=\pi k_{B} T /\left(\gamma \sigma^{2}\right)$ (full symbols) and the surface tension $\gamma$ in units of $k_{B} T_{c} / \sigma^{2}$ (open symbols), of the free liquid surface at temperatures above the triple point. Squares: $\phi_{\mathrm{LJ}}$; circles: $\phi_{\mathrm{Na}}$; triangles: $\phi_{\text {soft-1 }}$, rombhus: $\phi_{\text {repul }}$, and stars: $\phi_{\mathrm{Hg}}$.

plies an exponential dependence of the layering amplitude with the transverse size of the sampling box. ${ }^{5,27}$ The theoretical prediction, which had already been tested in a moleculardynamics simulation of a liquid-liquid interface of an inmiscible LJ binary mixture at low temperature and high pressure, ${ }^{28}$ is that the period and decay lengths of the oscillatory structures should be approximately independent of the transverse size $L_{x}$, but the amplitude of the oscillations should decay proportionally to $L_{x}^{-\eta}$, with an exponent approximately given by $\eta \approx \pi k_{B} T /\left(\gamma \sigma^{2}\right)$, in terms of the liquid-vapor surface tension $\gamma$ and the temperature.

In Fig. 11 (open symbols), we present the surface tension (in adimensional units $k_{B} T_{c} / \sigma^{2}$ ) for the $\mathrm{LJ}$ model and the different pair potentials used in this work. These results were obtained directly for the same slab-MC simulations as the density profiles in Fig. 10, and despite the different shapes of the pair potentials they all follow quite well a common trend with linear decay. This empirical corresponding-states law seems to indicate that the cold liquids (down to $T=0.15 T_{c}$ ), made accessible by the low $T_{t}$ in our models, are the natural extension of the usual simple liquid which have been studied at $T>0.5 T_{c}$, both experimentally and theoretically. The decay of $\gamma$ and the direct dependence of the exponent $\eta$ with temperature produce a rapid increase of its value, from $\eta$ $\simeq 0.29$ at $T_{t}$ for the $\phi_{\text {soft-1 }}$ potential to $\eta \approx 3.5$ at the triple point of the LJ model, and diverging as $T$ approaches $T_{c}$. The figure shows that the exponent $\eta$ as a function of $T / T_{c}$ takes similar values for all the different model potentials explored here, although with different ranges of accessibility imposed by the value of $T_{t}$ in each case. This may explain the qualitative similarity of the density profiles in Fig. 10 at the same reduced temperature for different model potentials, since we could assume that for any of these models a hypothetical sampling of the liquid-vapor interface over a transverse region of molecular size $L_{x} \approx \sigma$ would give an intrinsic density profile with strong layering structure (of amplitude similar to the bulk density). The predicted $\mathrm{CW}$ damping of the layering produces amplitudes of order $\left(L_{x} / \sigma\right)^{-\eta\left(T / T_{c}\right)}$ which have similar values for the different model potentials at the same reduced temperature.

The main implication of the above interpretation is that, even for the smallest value of $\eta \approx 0.29$ that we have obtained (and which is similar to the estimation from the experimental surface tension of $\mathrm{Hg}$ at the triple point) the amplitude of the density oscillations would depend strongly on the transverse size of the system. To have observable layering amplitudes, of a few percents of the bulk density, in computer simulations with the typical simulation sizes of $L_{x} \approx 10 \sigma$, requires to have a decay exponent $\eta$ of 1.2 or less, which (from the results in Fig. 10) implies $T / T_{c}<0.35$, well below the triple point for the LJ potential. However, a smaller transverse size, say $L_{x}=5 \sigma$ would increase that threshold to about $T / T_{c}$ $<0.5$, and for $L_{x}=3 \sigma$ the layering may start to appear at the LJ triple point. However, such small box-size simulations would lead to all kind of spurious effects from artificial correlations of the molecules with their images at the periodic boundary conditions.

The typical transverse size for the experimental observation of layering structures with $\mathrm{x}$-ray reflectivity have been estimated ${ }^{1,14}$ to be around $1000 \AA$, or $L_{x}=300 \sigma$ in terms of the atomic size, so that the temperature threshold for observing layering would be much lower than in our MC simulations. At the triple point temperature of $\mathrm{Hg}\left(T=-35^{\circ} \mathrm{C}\right.$, $T_{t} / T_{c} \approx 0.13$ and $\eta \approx 0.3$ ) the layering would already be damped to about $20 \%$ of the 'intrinsic' value, while at $T / T_{c}=0.2$ the damping would be nearly complete, leaving only about $3 \%$ of the intrinsic amplitude. This is in good agreement with the experimental observation ${ }^{1}$ that the strong peak over the Fresnel reflectivity observed at the triple point is already very weak, but still visible, at $T=23{ }^{\circ} \mathrm{C}\left(T / T_{c}\right.$ $=0.16$ ).

The strong dependence of the liquid-vapor density profiles in the transverse size sets a problem in the use of density-functional theoretical techniques to study structured density profiles, since these approaches have no explicit dependence on $L_{x}$. The qualitative similarity of our MC density profiles and those obtained with density-functional approximations for the square-well model, ${ }^{29}$ and for some liquid-metal models, ${ }^{11}$ support the general believe that these results correspond to effective values of $L_{x}$ similar to the correlation length in the bulk liquids (i.e., a few times $\sigma$ ). The oscillatory wavelength and the decay length of the layering structures would probably be well described by those density functional approximations; as they give good results for the location of the Fisher-Widom line. ${ }^{29}$ However, the incertitude on the precise value of the effective transverse size makes impossible any direct comparison of the oscillation amplitudes with simulations or experiments. Only in systems with extremely stiff fluid-fluid interfaces, like in the Gaussian overlap model ${ }^{30}$ for binary mixtures of immiscible polymers, there are reports of $\eta \approx 0.1$, which would make the $\mathrm{CW}$ damping effect weak enough for direct comparison of computer simulations and density-functional results.

The dependence of the layering amplitude with the reduced temperature $T / T_{c}$ and with the transverse size is very similar for all the model potentials studied here, so that the density profiles in Fig. 10 appear to be quite similar for $\phi_{\mathrm{Na}}$ and $\phi_{\mathrm{Hg}}$. However, these density profiles have some hidden clues on the different microscopic structure of the liquidvapor interface for these model interactions. Following a 


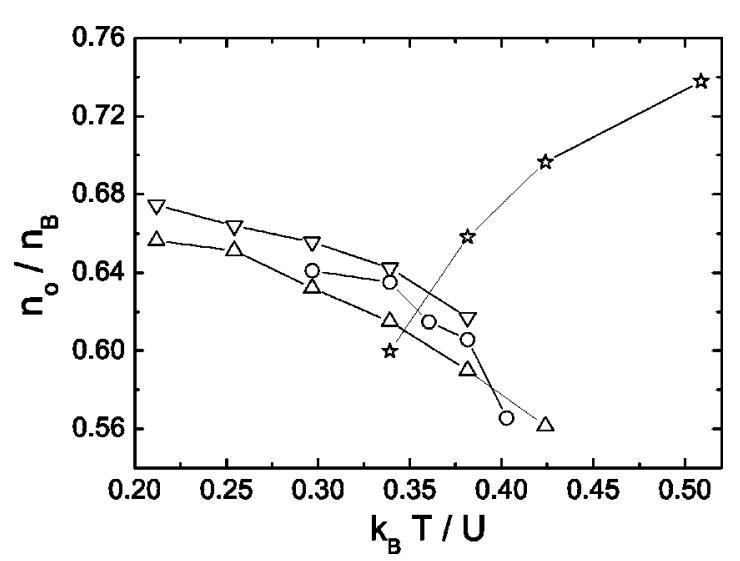

FIG. 12. Two-dimensional density of first layer, $n_{0} / n_{B}$, in the slab at temperatures above the melting point. Circles: $\phi_{\mathrm{Na}}$; up-triangles: $\phi_{\text {soft- } 1}$; downtriangles: $\phi_{\text {soft-2 }}$, and stars: $\phi_{\mathrm{Hg}}$.

standard procedure in the analysis of x-ray reflectivity experiments, ${ }^{31}$ we have fitted the density profiles as superposition of Gaussian layers:

$$
\rho(z)=\sum_{i=0}^{\infty} \frac{n_{i}}{\xi_{i} \sqrt{2 \pi}} \exp \left[-\frac{\left(z-z_{i}\right)^{2}}{2 \xi_{i}^{2}}\right] .
$$

The number of fitting parameters is usually reduced by using equally spaced Gaussians, located at $z_{i+1}=z_{i}+\Delta$, and assuming a linear increase of the squared width, $\xi_{i+1}^{2}=\xi_{i}^{2}$ $+\zeta$, and constant normalization $n_{i}=n_{B}$ for all the peaks beyond the second layer. The main information on the microscopic structure of the interface is given by the ratio $n_{0} / n_{B}$, i.e., the two-dimensional density of the first layer relative to the bulk density. The values of $n_{0} / n_{B}$ obtained from our MC simulations are shown in Fig. 12 as function of the temperature. All of them show first layers with lower coverage than the equivalent coverage at bulk density, and this is correlated with the general shape of the oscillating density profiles in Fig. 10, in which the first peak is clearly lower than the inner ones. The results for the model potential $\phi_{\mathrm{Na}}$ and its softcore variations, $\phi_{\text {soft-1 }}$ and $\phi_{\text {soft-2 }}$, give similar results with slightly decreasing coverage as $T$ increases. The model potential $\phi_{\mathrm{Hg}}$ gives the opposite result, with a rapid increase of $n_{0} / n_{B}$ with temperature. Unfortunately, the fit of the MC density profiles to the form (4) becomes ill-defined as the layering structure is damped below the intrinsic noise of the computer simulations, so that the microscopic information about the liquid-vapor interface which may be extracted in this way is limited to the low-temperature range. On the other hand, the results for $n_{0} / n_{B}$ should be independent of the $\mathrm{CW}$ broadening of the interface and the transverse size of the sampling, so that they may be directly compared with experiments and theoretical results.

The results from the fits to the x-ray reflectivity experiments on $\mathrm{Hg}$ and $\mathrm{Ga}$ show a quite different result, with values of $n_{0} / n_{B}>1$, which may reflect the effects of the metallic bond in these systems, neglected by our pair-potential model interactions. Nevertheless, the calculation of $\rho(z)$ from the raw experimental reflectivity data is subject to its own uncertainties, and there are theoretical predictions for the liquid metals ${ }^{32}$ showing a first layer clearly lower than the others. Results from density-functional calculations for the square-well fluid ${ }^{29}$ and simulations of layered fluid-fluid density profiles ${ }^{28}$ also present the same aspect as our density profiles, implying $n_{0} / n_{B}<1$, but apparently the opposite behavior is observed in the Gaussian-core model ${ }^{30}$ and for asymmetric hard-sphere binary mixtures ${ }^{33}$ at least for well developed oscillations.

\section{CONCLUSIONS}

The conclusions of our work cover two apparently different aspects: The existence of pairwise additive potential interactions with very low triple point temperature (down to $T_{t} / T_{c} \approx 0.15$ ) and the structure of the cold liquid-vapor interfaces in these models. The motivation for both aspects comes from the experimental results for some liquid metals, like $\mathrm{Hg}$ and $\mathrm{Ga}$, which have low melting points and show structured liquid surfaces, with strong molecular layers which become damped as the temperature increases. This work provides further evidence to confirm our hypothesis ${ }^{13}$ that these two aspects are closely linked, so that layering should be a common feature of free liquid surfaces well below the critical temperature, but that in simple molecular fluids it is is preempted by solidification, below which the liquid becomes metastable and the free liquid-vapor interface cannot be observed at equilibrium. The explanation for the origin of surface layering does not rely on the existence of nonadditive particle interactions, which have been invoked by various authors as a necessary requirement, but only on the thermodynamic stability of very cold liquids.

We have shown that very low triple-point temperatures may be achieved with realistic isotropic pairwise interactions, based on simplified treatments of different liquid metals $(\mathrm{Na}$ and $\mathrm{Hg})$ leading to potential functions $\phi(r)$ without kink, shoulders or double-well structures that might be regarded as spurious artificial features. The enhancement of some characteristics of these potentials make clear that there are at least two possible ways to get low values of $T_{t} / T_{c}$. The first one, emanating from the description of the alkali metals and enhanced in our model potential $\phi_{\text {soft-1 }}$, is a soft repulsive core, which from the fit of the Mayer function at the relevant temperatures may be described as similar to an inverse power $\phi(r) \sim r^{-n}$, with $n \approx 6.8$ for $\phi_{\mathrm{Na}}$ and $n \approx 4.6$ for $\phi_{\text {soft-1 }}$. Following the trend observed for such soft inverse-power cores, the density gap between liquid and solid become smaller and the atomic vibrations in the solid become larger than in systems with harder core repulsion. We have checked that at the triple-point temperature the most stable crystal structure for these models is the compact fcc, but at least for the $\phi_{\text {soft-1 }}$ potential a transition to the bcc at lower temperatures may appear.

The second mechanism to lower $T_{t} / T_{c}$ is provided by an inverse fit to the structure of liquid $\mathrm{Hg}$ which leads to a rather flat and broad potential well, with a particular width which destabilizes compact solid structures. The effect has been enhanced using the square-well model with an adequate choice of the well width, and it has shown to be quite effective in lowering the melting temperature, but with a behavior rather different from that observed in soft-core models. Atomic vibrations in the $\mathrm{Hg}$ model remain quite below those 
in the LJ model up to very close to the melting temperature where there is a rapid increase in the value of the Lindemann parameter (Fig. 8) with increasing $T$, associated to a rapid decrease of the equilibrium solid density at zero pressure (Fig. 3), but still leading to a quite large density gap with the liquid phase at the triple point. The systems with this kind of potential interactions may have stable solids with less symmetric crystalline structures that those we have checked here (fcc, hcp, bcc), as is the case for real $\mathrm{Hg}$, which crystallizes in a rhombohedral lattice. The MC simulations for the square-well fluid at low $T$ show a very slow equilibration, which may indicate the approach to glassy states or local crystallization in crystalline droplets. This slow equilibration of the square-well fluid for $\lambda=1.72$ has made it impossible for us to get a good estimate of $T_{t}$. Nevertheless, the study of the full crystal phase diagram for the different model potentials is beyond the scope of the present work, since our original motivation was the structure of the cold liquid, made stable by the lowering of the triple point, and its liquidvapor interface.

The usual range for the thermodynamic stability of liquids is about $0.5<T / T_{c}$, and our simple model interactions have pushed it down to $0.15<T / T_{c}$. The thermodynamic properties of such "cold" liquids seem to follow the trend of the usual "hot" liquids, both the bulk coexisting densities (Fig. 3) and the liquid-vapor surface tension (Fig. 11), suggesting an enlarged temperature range for a sort of corresponding-states law. With respect to the layering of the liquid surface, we conclude that it is indeed a generic behavior of cold liquids, but at the same time we have realized that the information given by the density profiles (like those in Fig. 10) is strongly dependent on the transverse size of the sampling. Any oscillatory density profile for liquid-vapor interfaces should be regarded as $\rho\left(x, L_{x}\right)$ since the amplitude of the oscillations would decay as $L_{x}^{-} \eta(T)$, with the exponent $\eta$ presented in Fig. 11 for our model potentials. This is certainly a problem in the comparison of computer simulations with experimental results (for which $L_{x}$ may be estimated but is not known with precision) and with density functional or other theoretical results (for which there no dependence on $L_{x}$ ). Nevertheless, there is a shortcut to this problem, which has been pioneered by the interpretation of the x-ray reflectivity results, ${ }^{31}$ in extracting the "intrinsic" features of the density profiles not affected by the capillary wave broadening of the interface and reflecting the truly microscopic structure of the liquid surfaces. We have followed that track by fitting the density profiles from our MC simulations to the form (4), and the results for the coverage of the first layer in Fig. 12 could be directly compared with the results of density-functional approximations, which would clarify its significance and its relationship with the molecular interactions. The challenge for the future is to extract and interpret the truly microscopic information which is hidden by the smoothing of the liquid-vapor density profile.

\section{ACKNOWLEDGMENT}

This work has been supported by the Dirección General de Educación Superior e Investigación Científica of Spain, under Grant Nos. BFM2001-1679-C03 and 1FD97-1358.

${ }^{1}$ O. Magnussen et al., Phys. Rev. Lett. 74, 4444 (1995); E. DiMasi et al., Phys. Rev. B 58, R13419 (1998).

${ }^{2}$ M. Regan et al., Phys. Rev. Lett. 75, 2498 (1995).

${ }^{3}$ B. Ocko et al., Phys. Rev. Lett. 57, 94 (1986).

${ }^{4}$ Y. Martínez-Ratón, A. Somoza, L. Mederos, and D. Sullivan, Faraday Discuss. 104, 111 (1996).

${ }^{5}$ R. Evans, in Fundamentals of Inhomogeneous Fluids, edited by D. Henderson (Dekker, New York, 1992), pp. 85-175.

${ }^{6}$ J. S. Rowlinson and B. Widom, Molecular Theory of Capillarity (Clarendon, Oxford, 1982).

${ }^{7}$ D. Beaglehole, in Fluid Interfacial Phenomena, edited by C. A. Croxton (Wiley, New York, 1986), pp. 523-554

${ }^{8}$ H. Tostmann et al., Phys. Rev. B 61, 7284 (2000).

${ }^{9}$ J. Harris, J. Gryko, and S. Rice, J. Chem. Phys. 87, 3069 (1987); M. Zhao, D. Chekmarev, Z. Cai, and S. Rice, Phys. Rev. E 56, 7033 (1997).

${ }^{10}$ F. Celestini, F. Ercolessi, and E. Tosatti, Phys. Rev. Lett. 78, 3153 (1997); S. Iarlori, P. Carnevali, F. Ercolessi, and E. Tosatti, Surf. Sci. 211/212, 55 (1989)

${ }^{11}$ M. A. Gómez and E. Chacón, Phys. Rev. B 46, 723 (1992); Phys. Rev. B 49, 11405 (1994); M. Hasegawa and M. Watabe, J. Phys. C 15, 353 (1982).

${ }^{12}$ G. Fabricius et al., Phys. Rev. B 60, R16283 (1999).

${ }^{13}$ E. Chacón, M. Reinaldo -Falagán, E. Velasco, and P. Tarazona, Phys. Rev. Lett. 87, 166101 (2001)

${ }^{14}$ P. Tarazona, E. Chacón, M. Reinaldo-Falagán, and E. Velasco, J. Chem. Phys. 117, 3941 (2002).

${ }^{15}$ Y. Li, E. Blaisten-Barojas, and D. A. Papaconstantopoulos, Phys. Rev. B 57, 15519 (1998).

${ }^{16}$ R. Kumaravadivel and R. Evans, J. Phys. C: Solid State Phys. 9, 3877 (1976).

${ }^{17}$ F. Hensel and W. W. Warren, Fluid Metals (Princeton University Press, Princeton, New Jersey, 1999).

${ }^{18}$ D. Frenkel and B. Smit, Understanding Molecular Simulation (Academic, New York, 1996).

${ }^{19}$ S. Munejiri, F. Shimojo, and K. Hoshino, J. Phys.: Condens. Matter 10, 4963 (1998)

${ }^{20}$ L. Vega, E. de Miguel, L. F. Rull, G. Jackson, and I. A. McLure J. Chem. Phys. 96, 2296 (1992); J. Chang and S. I. Sandler, Mol. Phys. 81, 745 (1994).

${ }^{21}$ D. A. Young and B. J. Alder, J. Chem. Phys. 73, 2430 (1980)

${ }^{22}$ P. Tarazona, Phys. Rev. Lett. 84, 694 (2000).

${ }^{23}$ F. A. Lindemann, Z. Phys. 11, 609 (1910).

${ }^{24}$ J. P. Hansen and I. R. McDonald, Theory of simple liquids (Academic, New York, 1976).

${ }^{25}$ J. P. Hansen and L. Verlet, Phys. Rev. 184, 768 (1969).

${ }^{26}$ R. Agrawal, and D. A. Kofke, Mol. Phys. 85, 23 (1995).

${ }^{27}$ R. Evans, in Les Houches, Session XLVIII, Liquids at Interfaces (Elsevier, New York, 1989).

${ }^{28}$ S. Toxvaerd and J. Stecki, J. Chem. Phys. 102, 7163 (1995).

${ }^{29}$ R. Evans, J. R. Henderson, D. C. Hoyle, A. O. Parry, and Z. A. Sabeur, Mol. Phys. 80, 755 (1993).

${ }^{30}$ A. J. Archer and R. Evans, Phys. Rev. E 64, 041501 (2001),

${ }^{31}$ Jeffrey Penfold, Rep. Prog. Phys. 64, 777 (2001).

${ }^{32}$ D. Chekmarev, M. Zhao, and S. A. Rice, J. Chem. Phys. 109, 768 (1998).

${ }^{33}$ J. M. Brader, R. Evans, M. Schmidt, and H. Lowen, J. Phys.: Condens. Matter 14, L1 (2002) 